\title{
A Novel Method for Modelling a Thermopile Infrared Sensor in PSpice
}

\author{
H.X. Chen \\ Department of Physics \\ Tong Hua Normal University \\ China
}

\author{
L.H. Xiang \\ Department of Physics \\ Northeast Normal University \\ China
}

\author{
L.M. Wang \\ Department of Physics \\ Northeast Normal University \\ China \\ *Corresponding author
}

\begin{abstract}
Presently, thermopile infrared sensors have been successfully commercialized in different application areas such as contactless temperature measurements and infrared detectors. In the process of designing the application circuit of thermopiles, circuit simulation is necessary to improve the efficiency of the circuit design. This work reports a method based on neural networks to model a thermopile infrared sensor, by which the fundamental characteristics including the temperature-voltage output characteristic, the frequency response characteristic, the angle characteristic and the optical characteristic are modelled in PSpice. Firstly, three appropriate neural networks are created and trained to approach each input-output characteristic respectively, after which the structures, weights and biases of each neural network are acquired. Secondly, the obtained structures are described with the PSpice programming language to establish sub-circuits respectively. Finally, these sub-circuits are integrated into a unitary circuit based on the relationship between the inputs and output of the sensor to form the final model.
\end{abstract}

\section{Keywords-neural network;PSpice;thermopile;model}

\section{INTRODUCTION}

Nowadays, the success of efficient and accurate circuit design should be attributed to varieties of simulation tools which are used to verify circuit designs and to predict the circuit behavior. Typically, PSpice is a powerful, generalpurpose analog and mixed-mode circuit simulator, which can do several types of circuit analyses. In addition, PSpice has plenty of libraries of standard analog and digital components such as diodes, transistors, flip-flops, and logic gates, which makes it a powerful circuit simulation tool. However, few sensor model libraries have been provided, which causes a problem that circuits with some sensors cannot be simulated as a whole in PSpice. The models of thermopile infrared sensors are not provided as well.

To date, existing sensor models are aimed either at describing the physical structures of sensors with mathematical formulas [1][2] for mathematical simulations, or simulating and designing sensors with the finite element models [3][4]. Other models rely on the physical model in which the energy domains of the sensors are described by means of electrical equivalents [5].

Neural network has been widely used in modelling some single-input and single-output (SISO) electronic devices and sensors such as NTC thermistors [6], Tunnel Diodes [7]. In this paper, we will extend the method to model a thermopile infrared sensor, TP336E, which has complex multi-input and single-output (MISO) characteristic, for transient analysis in PSpice. Of course, this method may be extended to model other thermopile infrared sensors easily.

\section{THERMOPILEOPERATING PRINCIPLE}

A thermopile, which is composed of thermocouples connected usually in series or less commonly in parallel, is a sensor converting thermal energy into electrical energy. The operating principle is Seebeck effect which is the conversion of temperature differences directly into electricity. Fig.1 (A) shows the Seebeck effect principle, where two chemically different materials $\mathrm{A}$ and $\mathrm{B}$ whose ends a (for hot thermo junction) and $b$ (for cold thermo junction) are welded to obtain a circuit. The $\mathrm{c}$ point is subjected to incident flux variations $(\mathrm{W} / \mathrm{m} 2)$ causing temperature to rise, which comply with the Blackbody Radiation Law [8][9].

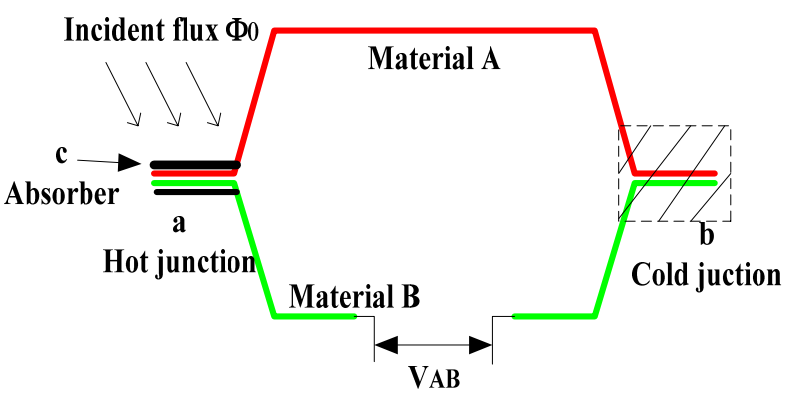




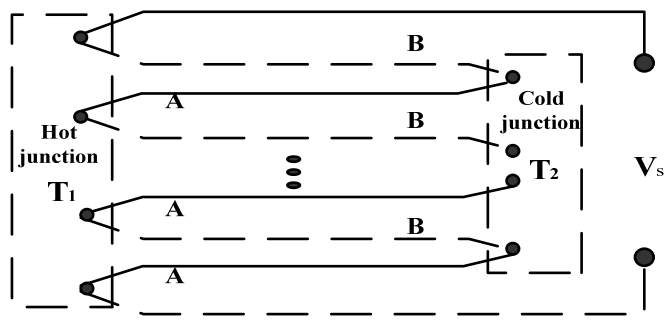

FIGURE I. (A) SEEBECK EFFECT PRINCIPLE, (B) STRUCTURE OF A THERMOPILE.

The temperature difference between two different materials causes a continuous current in the conductors if they form a complete loop. The voltage $V_{A B}$ is created several microvolts per Kelvin difference, which can be expressed as equation (1).

$$
V_{A B}=\left(S_{B}-S_{A}\right)\left(T_{2}-T_{1}\right)
$$

Where $S_{A}, S_{B}$ are the Seebeck coefficients of the metal A and $\mathrm{B}$, Which are effectively constant at the measured temperature range; $T_{1}$ and $T_{2}$ are the temperatures of the two junctions.

Thermocouples can be connected in series to form a thermopile, where all the hot junctions are exposed to a higher temperature and all the cold junctions to a lower temperature, as shown in Fig. 1(B). Thus, based on the total number of thermocouples $\mathrm{N}$, the total voltage $V_{S}$ generated by the thermopile can be written as:

$$
V_{S}=N \times V_{A B}
$$

TP336E consists of 116 thermocouples, whose output voltage depends on the temperature of the object measured, the angle of radiation, the chopper frequency and the parameter of the optical filter. The correspondingcharacteristic curves, temperature-voltage output characteristic, the frequency response characteristic,the angle characteristic and the optical characteristic, are shown in Fig.2.
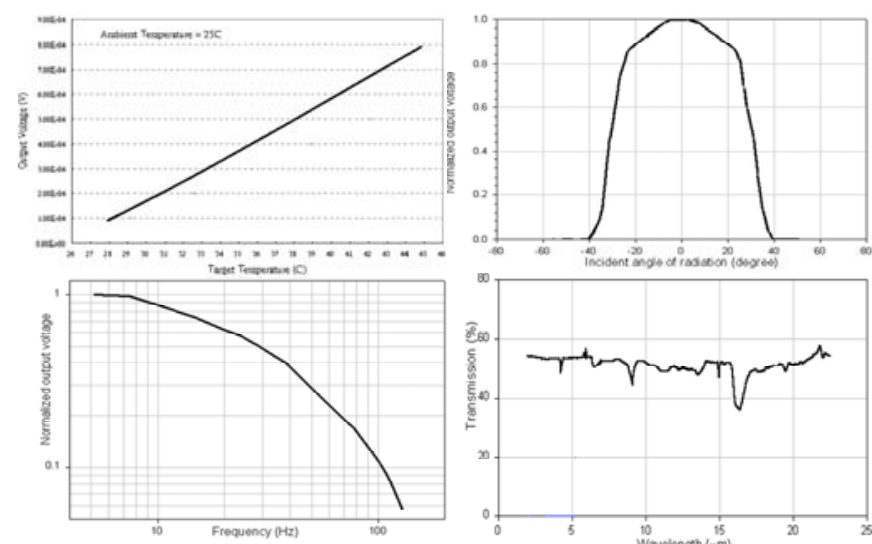

FIGURE II. THE CHARACTERISTIC CURVES OF TP336E.

\section{NEURAL NETWORK APPROACHING}

Since the output voltage of a thermopile is affected by those four factors discussed above, the expected model structure is described in Fig.3:

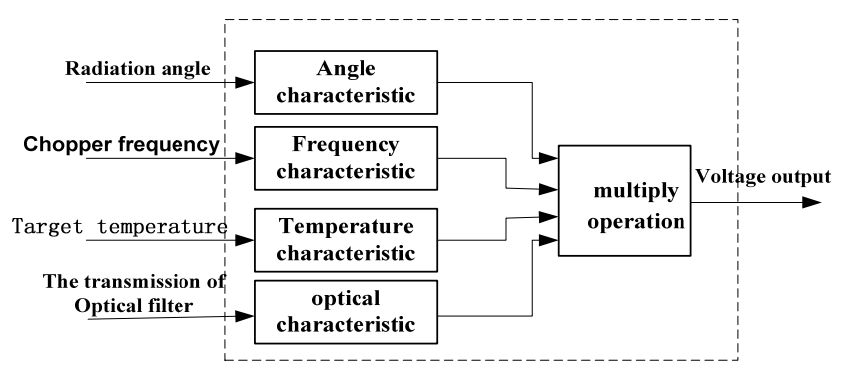

FIGURE III. EXPECTED MODEL STRUCTURE.

Undoubtedly, it is a complex MISO model. Although a MISOneural networkcan be applied to approximate the four characteristics, it is found that the structure is socomplexas to make a huge sub-circuit in PSpice. Therefore, we chose to construct the neural networks for the temperature-voltage output characteristic, the frequency response characteristic,the angle characteristic and the optical characteristic respectively, and then integrate them into a complete circuit.

\section{A. Approaching Temperature-Voltage Characteristic}

The relationship between the object temperature and the sensor output voltage is approximatively linear, so a twolayer feedforward neural network with linear activation function was chosen to approach it. The resulting network consists of one neuron in the hidden layer and one neuron in the output layer. The obtained structure and corresponding weights and biases are shown in Fig.4 and Table1 respectively.

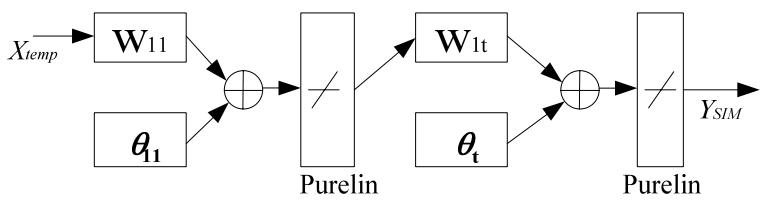

FIGURE IV. NEURAL NETWORK STRUCTURE FOR TEMPERATURE VOLTAGE CHARACTERISTIC.

TABLE I.WEIGHTS AND BIASES FOR TEMPERATURE VOLTAGE CHARACTERISTIC

\begin{tabular}{|c|c|c|c|c|}
\hline $\mathbf{j}$ & $\mathbf{W}_{\mathbf{1 j}}$ & $\boldsymbol{\theta}_{\mathbf{1 j}}$ & $\mathbf{W}_{\mathbf{j t}}$ & $\boldsymbol{\theta}_{\mathbf{t}}$ \\
\hline 1 & $-6.0483 \mathrm{e}-5$ & 0.4866 & 0.68426 & 0.33189 \\
\hline
\end{tabular}

\section{B. Neural Network Training For Frequency Response} Characteristic

According to the features of the frequency response characteristic, a SISO orthogonal polynomial neural network is created, whose activation functions of hidden layer neurons are a sequence of Chebyshev orthogonal polynomials as shown in equation (3). 


$$
\begin{aligned}
& T_{0}(x)=1 \\
& T_{1}(x)=x \\
& T_{n+1}(x)=2 x T_{n}-T_{n-1}(x), \quad n=1,2, \cdots
\end{aligned}
$$

The weights from the input to the hidden layer neurons are constant 1 , while the weights from the hidden layer neurons to the output are vectors $w_{i}$, and the biases of hidden layer neurons and output layer neurons are constant 0 . The structure of the orthogonal polynomial neural network is shown in Fig. 5.

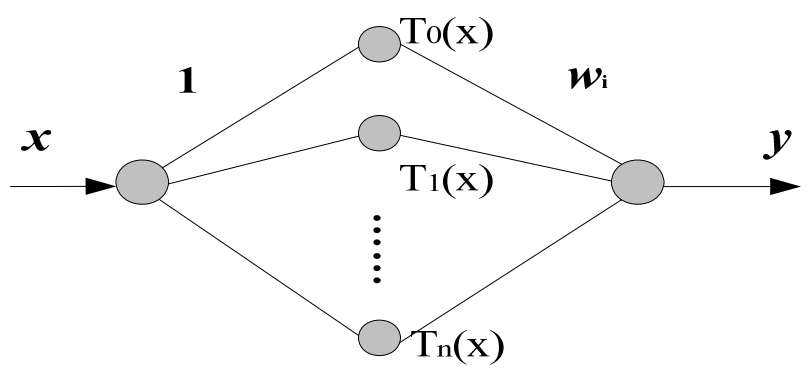

FIGURE V. ORTHOGONAL POLYNOMIAL NEURAL NETWORK STRUCTURE.

Since the input of Chebyshev orthogonal polynomial should range from 0 to 1 , the input data $\left(\mathrm{X}_{\text {fre }}\right)$ and target data $\left(\mathrm{V}_{\text {fre }}\right.$ ) of the frequency response characteristic have to be normalized by formula (4) firstly. Then, the normalized data $\mathrm{X}_{\text {fre_N }}$ and $\mathrm{V}_{\text {fre_N }}$ are used as the input and desired output of the neural network respectively.

$$
\begin{aligned}
X_{\text {fre_N }} & =\frac{X_{\text {fre }}-\min \left(X_{\text {fre }}\right)}{\max \left(X_{\text {fre }}\right)-\min \left(X_{\text {fre }}\right)} \\
V_{\text {fre_N }} & =\frac{V_{\text {fre }}-\min \left(V_{\text {fre }}\right)}{\max \left(V_{\text {fre }}\right)-\min \left(V_{\text {fre }}\right)}
\end{aligned}
$$

By applying the well-known BP algorithm, we obtained an optimal structure finally. The resulting two-layer network consists of five neurons in the hidden layer and one neuron in the output layer. The obtained weights and biases are shown in Table 2.

TABLE II.WEIGHTS AND BIASES FOR FREQUENCY RESPONSE CHARACTERISTIC.

\begin{tabular}{|c|c|c|c|c|c|}
\hline $\mathrm{i}$ & 1 & 2 & 3 & 4 & 5 \\
\hline $\mathrm{Wi}$ & 3.8810 & -6.0575 & 3.0302 & -0.9249 & 0.1260 \\
\hline
\end{tabular}

\section{Neural Network Training For Angle Characteristic}

In order to model the angle characteristic, we used the BP algorithm with updatingmomentum to train a SISO feedforward neural network to approach the curve. Since the activation function of the hidden layer is logarithmic sigmoid function, whose output is limited to the interval $(0$, 1 ), the sample data $V_{\text {angle }}$ should be normalized firstly.

By adjusting the network structure gradually, an optimal structure is achieved as shown in Fig.6, which contains sevenneurons with logsig activation functions in the hidden layer and one neuron with purelin activation function in the output layer.The weights and biases achieved are shown in Table 3.

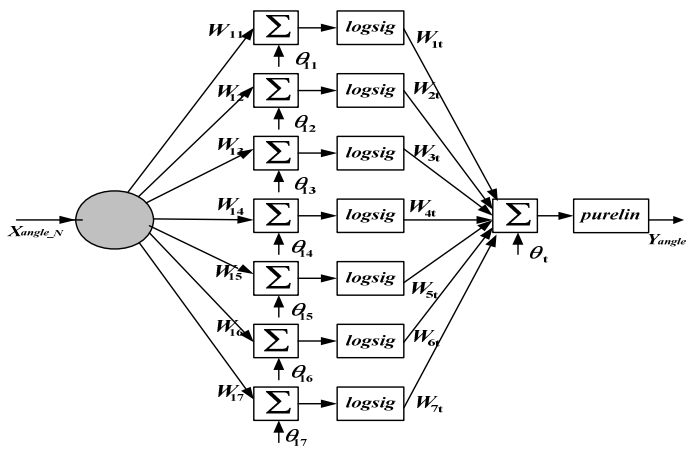

FIGURE VI. NEURAL NETWORK STRUCTURE FOR ANGLE CHARACTERISTIC.

TABLE III.WEIGHTS AND BIASES FOR ANGLE CHARACTERISTIC.

\begin{tabular}{|c|c|c|c|c|}
\hline $\mathrm{j}$ & $\mathrm{W} 1 \mathrm{j}$ & $\theta 1 \mathrm{j}$ & $\mathrm{Wjt}$ & $\theta \mathrm{t}$ \\
\hline 1 & 42.6524 & -39.3426 & 0.46286 & \\
\cline { 1 - 4 } 2 & -155.9305 & 142.4385 & 0.094693 & \\
\cline { 1 - 4 } 3 & -6.5478 & 2.5005 & 122.5607 & \multirow{2}{*}{0.19} \\
\cline { 1 - 4 } 4 & -82.5299 & 69.2656 & 0.24053 & \multirow{2}{*}{157} \\
\cline { 1 - 4 } 5 & -6.5791 & 2.5092 & -122.2882 & \\
\cline { 1 - 4 } 6 & -41.2983 & 3.6328 & -0.71312 & \\
\cline { 1 - 4 } 7 & 45.6607 & -7.5827 & 0.20742 & \\
\hline
\end{tabular}

IV. MODEL STRUCTURE DESCRIPTION IN PSPICE

To make the model applicable to circuit simulation in PSpice, the structure of the neural network should be described in PSpicecircuit description language. Activation functions are the most complex parts in the network, but it is easy to describe them in PSpice with Voltage Controlled Voltage Sources (E) by using the VALUE expression. Since the logsig function can be expressed as equation (5), it may be described with PSpice language as followed codes.

$$
\begin{aligned}
y_{j}= & \log \operatorname{sig}\left(\sum_{i=1}^{n} W_{i j} x_{i}-\theta_{j}\right)=1 / 1+e^{\left(\sum_{i=1}^{n} W_{i j} x_{i}-\theta_{j}\right)} \\
& \text { R2 } 101 \text { E9 }
\end{aligned}
$$

R3 210 1E9

EWB1 20 POLY(1) $(1,0)-\theta_{j} W_{i j}$

ELOG1 210 VALUE $=\{1 /(1+\operatorname{EXP}(-1 * \mathrm{~V}(2,0)))\}$

The following codes are for Chebyshev orthogonal polynomial.

\section{R4 10 1 $1 \mathrm{E} 9$}

\section{R5 $2101 \mathrm{E} 9$}

EWB 110 VALUE $=\{2 * \mathrm{~V}(1) * \mathrm{~V}($ node -1$)-\mathrm{V}($ node -2$)\}$ codes.

Purelin as equation (6) can be described with followed

$$
y_{t}=\text { purelin }\left(\sum_{j=1}^{p} W_{j t} y_{j}-\theta_{t}\right)=\sum_{j=1}^{p} W_{j t} y_{j}-\theta_{t}
$$

R6 60 1E9

$\mathrm{EF} 160 \mathrm{VALUE}=\left\{W_{1 t} * \mathrm{~V}(1)+W_{2 t} * \mathrm{~V}(2)+\cdots+W_{p t} * \mathrm{~V}(\mathrm{p})-\theta_{t}\right\}$

\section{THE UNITARY MODEL}

Till now, we have acquired corresponding sub-models for the temperature-voltage output characteristic, the frequency 
response characteristic andthe angle characteristic. Since the optical characteristic is nearly a constant, its transfer function may be modelled as an invariable transmission coefficient, whereby the fluctuations are ignored. To integrate the submodels into a complete model, a multiplying operation circuit is applied to form the final output, since the output of thermopileinfraredsensors is the product of the outputs of each characteristic theoretically. It should be noted that the structure of the final model shown in Fig. 3 consists of four inputs terminals, angle, chopper frequency, target temperature and the transmission of optical filter, and one output terminal. We can describe the structure in PSpicebythe following code.

\section{XOP 13 FREQUENCY \\ XOP1 24 ANGLE \\ XOP2 56 TEMPERATURE \\ $\mathrm{E} 1078$ VALUE $=\{\mathrm{V}(3) * \mathrm{~V}(4) * \mathrm{~V}(6) *$ Trans $\}$}

Where the variable Transrepresents thetransmission coefficient of the optical filter, which is $55 \%$ for TP $336 \mathrm{E}$.

\section{MODEL VALIDATION}

In order to verify the availability of the achieved model, a simulation test is practiced by performing DC Sweep Analysis in PSpice, during which the sweeping voltage representing the range of the incident angle is from -40deg to $40 \mathrm{deg}$, the range of target temperature is from $25^{\circ} \mathrm{C}$ to $50^{\circ} \mathrm{C}$, the range of chopper frequency is from $0 \mathrm{~Hz}$ to $128 \mathrm{~Hz}$ and the transmission coefficient of filter is 0.55 . The analysis result is shown in a three-dimensional plot in Fig.7 (A), where X-axis is the incident angle vector, Y-axis is the temperature vector, and the $\mathrm{Z}$-axis is the output voltage vector. The chopper frequency is made to be a constant $1 \mathrm{~Hz}$ during simulation.Fig.7 (B) shows another three-dimensional plotwhich is formed by the original data of the sensor and with same configurations as the Fig.7 (A). The comparison between the two figures shows that they are well-matched. The testing program is given below.

Test TP336E

.Lib modelTP336E.lib

Xtp336E 120 VOUT CURRENTS

Vtempreture 1001

Vangle 2001

Vfrequency 300 DC 1

.DC Vtempreture 26460.5

.DC Vangle -80801

.END

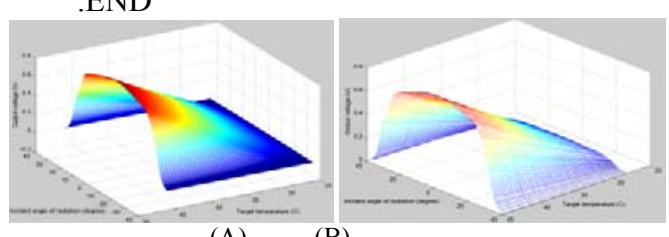

(A)

(B)

FIGURE VII. THE THREE-DIMENSIONAL PLOT OF (A)SIMULATION TEST (B) ORIGINAL DATA.

\section{CONCLUSIONS AND DISCUSSIONS}

An approach based on neural networks to model the thermopile infrared sensor with MISO characteristics in PSpice is presented in this paper. It is an expansion of the neural network method which has been applied to model SISO sensors.

The complexity of the created model depends primarily on that of the neural network used to approach the characteristic data of sensors. Therefore, the emphasis of our further researches will focus on exploring effective algorithms for generating simplest neural network structure.

The proposed modelling method in this paper can be extended to model other MISO sensors easily.

\section{ACKNOWLEDGEMENTS}

This work is supported by the Natural Science Foundation of Science and Technology Department of Jilin Province(20130102028JC).

\section{REFERENCES}

[1] J. F. Creemer, P. J. French, A new model of the effect of mechanical stress on the saturation current of bipolar transistors , Sensors and Actuators A: Physical, 97(4), pp. 289-2951, 2002.

[2] W. J. Wang, R. M. Lin, Q. B. Zou and X. X. Li,Modeling and characterization of a siliconcondenser microphone, Journal of Micromechanics And Microemgineering,14, pp.403-409, 2004.

[3] TimoVeijola, Thierry Corman, Peter Enoksson, Goran Stemme, Dynamic simulation model for a vibrating fluid density sensor, Sensors and Actuators A: Physical. 76(1), pp. 213-224, 1999.

[4] P. Bruschi, A. Ciomei, M. Piotto,Design and analysis of integrated flow sensors by means of a two-dimensional finite element model, Sensors and Actuators A. 142(1), pp. 153-159, 2008.

[5] Gerhard Fischerauer, Franz L. Dickert, An analytic model of the dynamic response of mass-sensitive chemical sensors, Sensor and Actuators B, 123(2),pp. 993-1001, 2007.

[6] L.M. Wang, Y.F. Deng, X.L. Zhao, and B.L.Liu,A neural network approach for creating a NTC thermistor model library for PSpice, IEEE International conference on Cybernetics and Intelligent Systerms, pp.1133-1137, 2008.

[7] Y. Huang, L.M. Wang, Neural network method for modellingnonlinear electron devices, Chinese Journal of Electron Devices, 28(4), pp.890-892, 2005.

[8] Thermocouple,http://en.wikipedia.org/wiki/Thermocouple.

[9] Kimmo Keraena, JukkaT.Mainena, PenttiKorhonena, EveliinaJuntunena,VeliHeikkinena, JakkeMaelab, Infrared temperature sensor system for mobile devices, Sensors and Actuators A, 158(1), pp.161-167,2010. 